\title{
Effects of Mucuna pruriens on Free Fatty Acid Levels and Histopathological Changes in the Brains of Rats Fed a High Fructose Diet
}

\author{
Bekir Akgun ${ }^{\mathrm{a}}$ Aysel Sarı ${ }^{\mathrm{b}}$ Sait Ozturk ${ }^{\mathrm{a}}$ Fatih Serhat Erol $^{\mathrm{a}}$ \\ Ibrahim Hanifi Ozercan ${ }^{c}$ Ramazan Ulu ${ }^{d}$ \\ Departments of ${ }^{\text {a }}$ Neurosurgery, ${ }^{b}$ Chemistry/Biochemistry, ${ }^{c}$ Clinical Pathology and ${ }^{d}$ Internal Medicine, \\ Firat University, Elazig, Turkey
}

\section{Significance of the Study}

- In this study, a high fructose diet fed to rats increased the levels of free arachidonic acid and docosahexaenoic acid, and also caused extensive gliosis, neuronal hydropic degeneration, and edema but these effects were reversed when Mucuna pruriens was added to the high fructose diet. Hence, M. pruriens could have a therapeutic benefit by reversing pathological changes caused by altered brain fatty acid metabolism and histopathological changes due to a high fructose diet.

\section{Keywords}

Brain · Free fatty acids · Fructose $\cdot$ Histopathology $\cdot$ Mucuna pruriens

\begin{abstract}
Objective: To investigate free fatty acid levels and histopathological changes in the brain of rats fed a high fructose diet (HFrD) and to evaluate the effects of Mucuna pruriens, known to have antidiabetic activity, on these changes. Materials and Methods: The study comprised 28 mature female Wistar rats. The rats were divided into 4 groups, each included 7 rats. Group 1: control; group 2: fed an HFrD; group 3: fed normal rat chow and $M$. pruriens; group 4: fed an HFrD and M. pruriens for 6 weeks. At the end of 6 weeks, the rats were decapitated, blood and brain tissues were obtained. Serum glucose and triglyceride levels were measured. Free fatty
\end{abstract}

\section{KARGER}

E-Mail karger@karger.com www.karger.com/mpp

\section{The Author(s) \\ Published by S. Karger AG, Basel \\ Karger \\ Open access}

This is an Open Access article licensed under the Creative Commons Attribution-NonCommercial-4.0 International License (CC BY-NC) (http://www.karger.com/Services/OpenAccessLicense), applicable to the online version of the article only. Usage and distribution for commercial purposes requires written permission. acid levels were measured in 1 cerebral hemisphere of each rat and histopathological changes in the other. The MannWhitney $U$ test was used to compare quantitative continuous data between 2 independent groups, and the KruskalWallis test was used to compare quantitative continuous data between more than 2 independent groups. Results: Arachidonic acid and docosahexaenoic acid levels were significantly higher in group 2 than in group $1(p<0.05)$. Free arachidonic acid and docosahexaenoic acid levels in group 4 were significantly less than in group $2(p<0.05)$. Histopathological examination of group 2 revealed extensive gliosis, neuronal hydropic degeneration, and edema. In group 4, gliosis was much lighter than in group 2, and edema was not observed. Neuronal structures in group 4 were similar to those in group 1. Conclusions: The HFrD increased the levels of free arachidonic acid and docosahexaenoic acid probably due to membrane degradation resulting from possible oxi- 
dative stress and inflammation in the brain. The HFrD also caused extensive gliosis, neuronal hydropic degeneration, and edema. Hence, M. pruriens could have therapeutic effects on free fatty acid metabolism and local inflammatory responses in the brains of rats fed an HFrD.

(C) 2017 The Author(s)

Published by S. Karger AG, Basel

\section{Introduction}

The dietary intake of excess fructose has negative effects on many tissues and may cause various metabolic disorders [1]. It has been shown that short-term feeding of rats with a high fructose diet (HFrD) leads to metabolic changes that may cause prediabetes in humans and in the case of longer HFrD exposure may result in type II diabetes [2]. A high carbohydrate diet is associated with metabolic syndrome, central obesity, hyperlipidemia, hyperglycemia, atherosclerosis, nonalcoholic fatty liver, endothelial dysfunction, insulin resistance, and hypertension [3-5]. Problems such as hypertension, stroke, renal insufficiency, cardiovascular morbidity, and high mortality could also develop as a result of these conditions $[5,6]$. In addition, several histological and clinical pathologies related to both proinflammation and increased oxidative stress in the brain have been demonstrated [7-9]. Cognitive decline, mood disorders, and behavioral abnormalities can develop due to increased cytokine production and brain lipid metabolism changes $[7,10]$.

Brain lipid metabolism is altered by high fructose or glucose intake $[7,11,12]$. A rat model of intracerebroventricular streptozotocin-induced brain insulin resistance showed decreased cerebral cortex concentrations of ethanolamine glycerophospholipid and phosphatidylserine, and increased concentrations of unesterified palmitate, stearate, and arachidonic acid (AA), suggesting increased phospholipase $\mathrm{A}_{2}$-mediated membrane degradation [7, 11]. Brain phospholipid concentration was reported to be reduced in a genetic mouse model of diabetes [12]. Increased hippocampal malondialdehyde concentration (a marker of polyunsaturated fatty acid and oxidative degradation, was reported in the hippocampus of genetically obese and hypertensive rats [7]. These findings indicate that the metabolism of brain free fatty acids (FFA) could be affected by these metabolic changes.

Mucuna pruriens is a legume grown in Africa, South America, and South Asia [13, 14]. M. pruriens has a positive effect on enzymes involved in carbohydrate metabolism, either by increasing insulin secretion or by acting as insulin thereby reducing blood sugar levels [13]. Antiinflammatory, hypolipidemic, and antithrombotic effects have also been reported [14]. M. pruriens has antioxidant and cytotoxicity-reducing effects, and its effect on neurodegenerative diseases such as Alzheimer and Parkinson diseases has been investigated $[15,16]$.

The roles of $M$. pruriens in preventing metabolic changes that can occur in rats fed an HFrD, and the effects of $M$. pruriens on the brain are not well understood. In this study, changes in FFA and histopathological changes in brain tissue were investigated in rats fed an HFrD, and the effects of $M$. pruriens on these changes were analyzed.

\section{Materials and Methods}

\section{Animals and Study Groups}

This study was approved by the Institutional Animal Research Ethics Committee. The Experimental Animals Implementation and Research Center supplied 28 mature female Wistar rats (aged 8-9 weeks) with normal motor activity and weighing 200-250 g for this study. The rats were housed in standard laboratory conditions (12-h light/dark cycle; room temperature $20-22^{\circ} \mathrm{C}$ ).

The rats were divided into 4 groups, each containing 7 rats. Group 1 (control): fed a normal rat chow diet and normal drinking water for 6 weeks; group 2 (HFrD): fed a normal rat chow diet and fructose water ( $50 \mathrm{~g}$ fructose powder per $100 \mathrm{~mL}$ drinking water) for 6 weeks; group 3 (M. pruriens): fed a normal rat chow diet and normal drinking water and given $100 \mathrm{mg} / \mathrm{kg} M$. pruriens every day by gavage for 6 weeks; group $4(\mathrm{HFrD}+M$. pruriens): fed a normal rat chow diet and fructose water $(50 \mathrm{~g}$ fructose powder per $100 \mathrm{~mL}$ drinking water) along with $100 \mathrm{mg} / \mathrm{kg} M$. pruriens every day by gavage for 6 weeks. M. pruriens dry extract was provided by OmniActive Health Technologies (item code: 51002; Hinjewadi, Pune, India). The extract was from $M$. pruriens dried seeds.

At the end of 6 weeks, blood samples were taken from the rats' tails just before decapitation. The samples were centrifuged at $3,000 \mathrm{~g}$ for $10 \mathrm{~min}$, sera decanted and stored at $-80^{\circ} \mathrm{C}$ until analysis. Serum glucose and triglyceride levels were measured with enzymatic assay kits (ADVIA Chemistry), by blood analyzer device (Autoanalyzer, Siemens). Brain tissues were taken and the whole brains were divided into 2 hemispheres. FFA levels were measured using gas chromatography (GC) using a Shimadzu GC instrument in 1 hemisphere. Histopathological examinations were performed, using light microscopy by a pathologist (I.H.O.) and a neurosurgeon (B.A.), in the other hemisphere.

\section{Preparation of Fatty Acid Methyl Esters}

Lipid extraction from tissue samples was performed using the Hara and Radin method, which uses a 3:2 volume/volume (v/v) hexane/isopropranolol mixture [17]. The fatty acids in lipids were converted to methyl ester derivatives for gas chromatographic analysis. To prepare the methyl ester derivaties [18], the lipid extract in the hexane/isopropranolol phase was transferred into 30 $\mathrm{mL}$ test tubes. Five milliliters of $2 \%$ methanol sulfuric acid was added and mixed well with a vortex. This mixture was left for methylation in the incubator at $50^{\circ} \mathrm{C}$ for $15 \mathrm{~h}$. The tubes were re- 
Table 1. Serum glucose and triglyceride levels (mg/dL)

\begin{tabular}{|c|c|c|c|c|c|c|c|c|}
\hline & \multirow{2}{*}{$\begin{array}{l}\text { Group } 1 \\
\text { (control) }\end{array}$} & \multirow{2}{*}{$\begin{array}{l}\text { Group } 2 \\
\text { (HFrD) }\end{array}$} & \multirow{2}{*}{$\begin{array}{l}\text { Group } 3 \\
\text { (M. pruriens) }\end{array}$} & \multirow{2}{*}{$\begin{array}{l}\text { Group } 4 \\
\text { (M. pruriens + } \\
\text { HFrD) }\end{array}$} & \multicolumn{4}{|l|}{$p$ values } \\
\hline & & & & & G1 vs. G2 & G1 vs. G3 & G1 vs. G4 & $\mathrm{G} 2$ vs. G4 \\
\hline Glucose & $85.7 \pm 10.9$ & $113.2 \pm 6.4$ & $78.7 \pm 8.9$ & $92.4 \pm 7.3$ & 0.002 & 0.22 & 0.21 & 0.004 \\
\hline Triglyceride & $90.4 \pm 24.9$ & $175.2 \pm 34$ & $88.8 \pm 11.3$ & $97.5 \pm 46.5$ & 0.001 & 0.43 & 0.32 & 0.003 \\
\hline
\end{tabular}

Table 2. Percentages of free fatty acid peaking in the brain tissue

\begin{tabular}{|c|c|c|c|c|c|c|}
\hline $\begin{array}{l}\text { Fatty acids } \\
\text { C:D }\end{array}$ & Group 1 & Group 2 & Group 3 & Group 4 & \multicolumn{2}{|l|}{$p$ values } \\
\hline $15: 0$ & $0.316 \pm 0.059$ & $0.241 \pm 0.078$ & $0.320 \pm 0.108$ & $0.328 \pm 0.163$ & $>0.05$ & $>0.05$ \\
\hline $16: 1$ & $0.456 \pm 0.207$ & $0.374 \pm 0.065$ & $0.339 \pm 0.127$ & $0.491 \pm 0.126$ & $>0.05$ & $>0.05$ \\
\hline $16: 0$ & $31.414 \pm 2.046$ & $30.754 \pm 1.192$ & $31.032 \pm 2.777$ & $29.783 \pm 3.399$ & $>0.05$ & $>0.05$ \\
\hline $18: 1$ & $17.583 \pm 1.966$ & $17.250 \pm 1.565$ & $17.269 \pm 1.826$ & $17.509 \pm 2.047$ & $>0.05$ & $>0.05$ \\
\hline 18:0 & $29.878 \pm 1.481$ & $29.321 \pm 2.959$ & $30.164 \pm 1.744$ & $29.693 \pm 3.748$ & $>0.05$ & $>0.05$ \\
\hline $20: 4$ & $6.247 \pm 0.811$ & $9.860 \pm 0.945$ & $6.043 \pm 0.961$ & $7.046 \pm 4.059$ & 0.002 & 0.01 \\
\hline $20: 0$ & $1.029 \pm 0.384$ & $0.616 \pm 0.111$ & $1.078 \pm 0.368$ & $1.208 \pm 0.284$ & $>0.05$ & $>0.05$ \\
\hline 21:0 & $2.926 \pm 0.702$ & $2.440 \pm 0.373$ & $2.827 \pm 0.399$ & $3.081 \pm 0.419$ & $>0.05$ & $>0.05$ \\
\hline $22: 6$ & $6.392 \pm 1.193$ & $8.887 \pm 1.033$ & $5.918 \pm 1.063$ & $6.839 \pm 1.050$ & 0.004 & 0.005 \\
\hline $24: 0$ & $0.376 \pm 0.297$ & $0.285 \pm 0.109$ & $0.226 \pm 0.054$ & $0.304 \pm 0.138$ & $>0.05$ & $>0.05$ \\
\hline
\end{tabular}

C, number of carbon atoms; D, number of double bonds of the molecule.

moved from the incubator, cooled to room temperature and mixed well by adding $5 \mathrm{~mL}$ of $5 \%$ sodium chloride. Fatty acid methyl esters were extracted with $5 \mathrm{~mL}$ hexane, and the hexane phase was placed into the pipette, treated with $2 \%$ potassium bicarbonate $\left(\mathrm{KHCO}_{3}\right)$ and kept for $4 \mathrm{~h}$ to allow the phases to separate. The solution of the mixture that contained methyl esters was then subjected to evaporation at $45^{\circ} \mathrm{C}$ and under nitrogen flow dissolved with $1 \mathrm{~mL}$ of hexane, placed into 2 -mL autosampler-covered vials, and analyzed with GC.

Gas chromatographic processes of fatty acid methyl esters were analyzed with a Shimadzu GC instrument. For this analysis, a Machery-Nagel capillary column was used. The temperature of the column was kept at $120-220^{\circ} \mathrm{C}$, the injection temperature was set to $240^{\circ} \mathrm{C}$; the detector temperature was kept at $280^{\circ} \mathrm{C}$. Nitrogen was used as the carrier gas. The mixture belonging to the standard fatty acid methyl esters was injected, then the fatty acid methyl esters of the samples, and the retention time of each fatty acid was determined. Acetone was used for preparing injection probes. After these procedures, analysis of the fatty acid methyl esters of the samples was performed. The results were determined as the percentage amount for each fatty acid among the fatty acids.

\section{Histopathological Examination}

For histopathological investigation, brain samples were fixed in neutral-buffered $10 \%$ formalin. Fixed tissue samples were embedded in paraffin and horizontally sectioned into $4-\mu \mathrm{m}$ slices with a microtome. After deparaffinization, sections were mounted on slides and stained with hematoxylin and eosin. The slides were assessed under a light microscope.

\section{Statistical Analysis}

The data were analyzed using SPSS (Statistical Package for Social Sciences for Windows v.22.0; SPSS Inc., Chicago, IL, USA). Means and standard deviations were used as descriptive statistical methods. The Mann-Whitney $U$ test was used to compare quantitative continuous data between 2 independent groups and the Kruskal-Wallis test was used to compare quantitative continuous data between more than 2 independent groups. The Mann-Whitney $U$ test was used as a complement to determine the differences after the Kruskal-Wallis $\mathrm{H}$ test. The findings were evaluated at the $95 \%$ confidence interval and at the 5\% significance level. $p<0.05$ was considered statistically significant. 

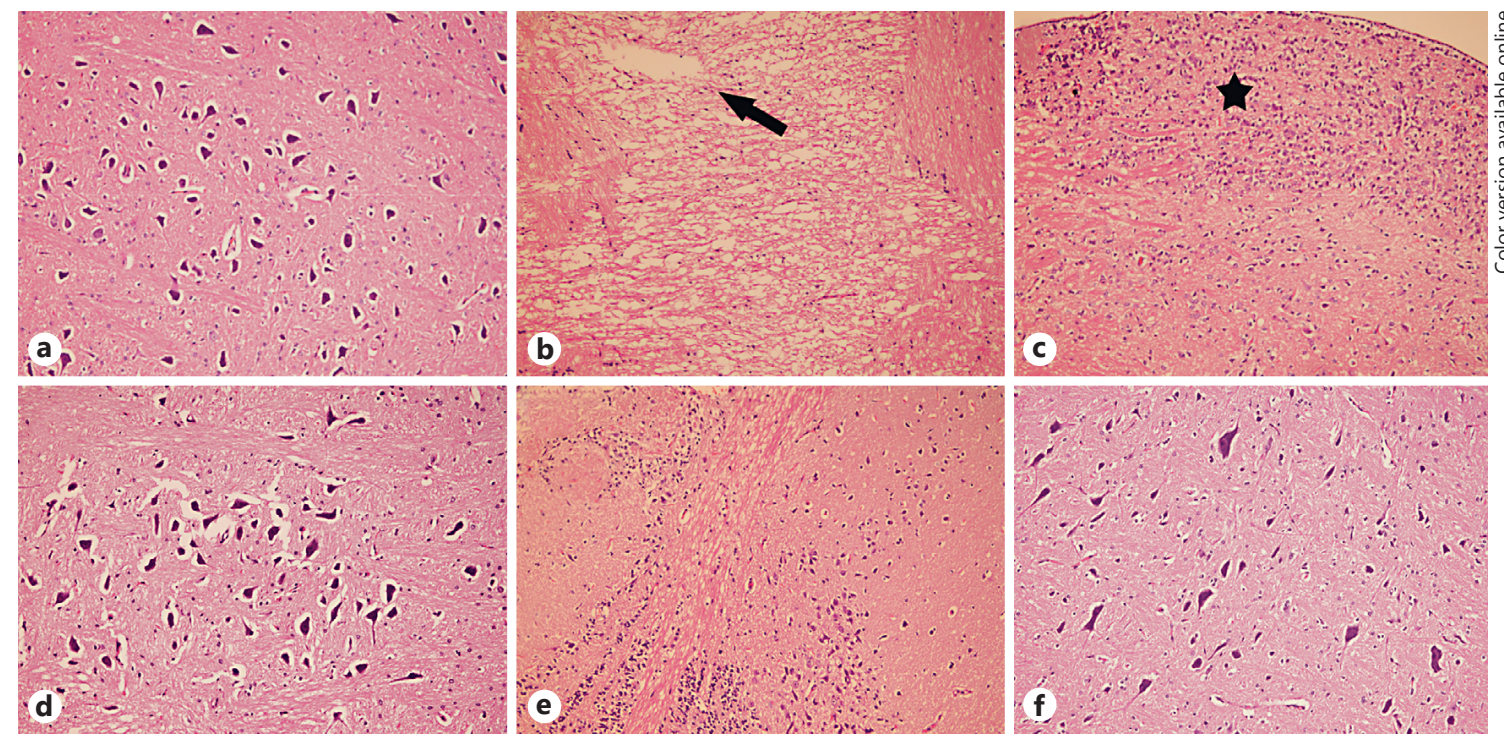

Fig. 1. a-f Histopathological representations of brain tissue (supratentorial region, gray matter-white matter interface) samples of rats. Hematoxylin-eosin. $\times 20$. a Normal neuronal structures are shown in group 1 . b Hydropic degeneration and edema (arrow) are seen in group 2. c Widespread gliosis (star) is also present in group 2. d Neuronal structures in group 3 are similar to group 1. e Slight gliosis is seen in group 4 . $\mathbf{f}$ Neuronal structures in group 4 are similar to groups 1 and 3.

\section{Results}

\section{Blood Tests}

The levels of serum glucose and triglyceride are presented in Table 1. Serum glucose and triglyceride levels were significantly higher in group 2 (glucose: $113.2 \pm 6.4$, triglyceride: $175.2 \pm 34$ ) compared with group 1 (glucose: $85.7 \pm 10.9$, triglyceride: $90.4 \pm 24.9)(p<0.05)$. Glucose and triglyceride levels were slightly higher in group 4 (glucose: $92.4 \pm 7.3$, triglyceride: $97.5 \pm 46.5$ ) than group 1 , but the difference was not statistically significant $(p>$ 0.05 ). Serum glucose and triglyceride levels were significantly less in group 4 compared with group 2 (M. pruriens) $(p<0.05)$.

\section{Brain Tissue FFA Levels}

The peak levels of all FFA in brain tissue by group are shown in Table 2. AA (20:4n-6) and docosahexaenoic acid (DHA) (22: 6n-3) levels were significantly higher $(p<0.05)$ in group 2 (AA: $9.860 \pm 0.945$, DHA: $8.887 \pm$ 1.033) than group 1 (AA: $6.247 \pm 0.811$, DHA: $6.392 \pm$ 1.193). Free AA and DHA levels were significantly less in group 4 (AA: $7.046 \pm 4.059$, DHA: $6.839 \pm 1.050)$ compared with group $2(p<0.05)$.

\section{Histopathology}

Normal neuronal structures were observed in group 1. Extensive gliosis, neuronal hydropic degeneration and edema were seen in group 2. Normal neuronal structures, similar to the control group, were also seen in group 3. In group 4, mild gliosis was seen, but edema was not observed and the neuronal structures were similar to those of group 1 . The histopathological features of the 4 groups are given in Figure 1.

\section{Discussion}

In this study, HFrD did not alter saturated or monounsaturated FFA levels, but higher levels of 2 specific polyunsaturated fatty acids, AA and DHA. These findings could lead to impairment of membrane integrity due to lipid peroxidation, as membrane degradation may increase the amount of free AA and DHA. Rats that were fed HFrD and received M. pruriens showed a significant decrease in free AA and DHA levels compared to rats fed with $\mathrm{HFrD}$ without $M$. pruriens. These findings indicate that $M$. pruriens might have an inhibitory effect on membrane degradation in the brain that can develop with an 
HFrD. Recent studies showed a significant expression of genes necessary for fructose metabolism in the brain [19]. It was clear from these data that several regions of the brain, including the cerebellum, hippocampus, cerebral cortex, and olfactory bulb, were capable of significant fructose metabolism. Additionally, this fructose metabolism was enhanced in brains after exposure to high fructose in the diet. Dietary fructose can induce some changes in brain metabolism, which could lead to possible detrimental metabolic dysregulation in the brain of individuals with a high fructose diet, as is seen in other organs [19].

Serum glucose and triglyceride levels increased significantly due to HFrD in our experiment. A high carbohydrate diet, also called a ketogenic diet, is known to cause a variety of metabolic diseases. Insulin resistance and elevated serum triglycerides are impacted by high levels of fructose in the diet. Elevated serum triglycerides have been postulated to disrupt insulin signaling by accumulating inside the cell [8]. Examination of the effects of such a diet on neurological diseases indicates that neurodegenerative disorders (e.g., Alzheimer and Parkinson diseases) may develop due to mitochondrial and cell membrane disorders. In addition, cognitive and behavioral changes may occur due to oxidative stress and inflammatory cytokine release $[7,10,20]$. Increased oxidative stress due to high fructose uptake enhances lipid peroxidation [9]. Changes in AA and DHA metabolism, which have important roles in cell membrane integrity and fluidity, have been reported in the brain due to membrane peroxidation $[7,11,21]$.

The finding of this study that rats fed an HFrD revealed extensive gliosis, neuronal hydropic degeneration, and edema in the brains could be due to possible oxidative stress and local inflammatory responses depending on inflammation. This finding could be due to HFrD and $M$. pruriens together causing gliosis that was much lighter, edema was not observed, and neuronal structures were similar to those of the control group. These indicate that M. pruriens could protect the brain against HFrD-related histopathological changes. However, several studies [22, 23] have shown that high blood sugar led to histopathological changes correlated with neuronal damage via oxidative stress and inflammatory cytokine release. Reactive gliosis is strongly associated with brain inflammation; increased inflammation is a key physiological feature of obesity, diabetes mellitus and/or metabolic syndrome [24]. The brain is highly sensitive to inflammation and inflammatory mediators, and metabolic dysfunction in both humans and laboratory animals has been associated with reactive gliosis and increased brain inflammation $[25,26]$. Probable effects of $M$. pruriens that might be responsible for the prevention of membrane degradation, gliosis, hydropic degeneration, and edema in the brain have been reported previously $[15,16]$. M. pruriens can reduce blood sugar levels by increasing insulin secretion or acting like insulin. Thus, it could prevent the development of insulin resistance in tissues. Also, M. pruriens could strengthen membrane stabilization by reducing inflammation through hypolipidemic, antithrombotic, antioxidant, and cytotoxicity-reducing effects. Some of the potential active components or fractions of $M$. pruriens may be responsible for the different effects $[13,16]$. Majekodunmi et al. [13] showed that the antidiabetic activity of $M$. pruriens seeds resides in the methanolic and ethanolic fractions of the extract. $M$. pruriens contains L-dopa, so its anti-Parkinson effects were attributed to its L-dopa content. Also M. pruriens seeds are rich in novel alkaloids, saponin, sterols, and minerals such as $\mathrm{Mg}^{2+}, \mathrm{K}^{+}$ and $\mathrm{Fe}^{3+}[13,16]$. The main limitation of this study included nonidentification of which components were essentially responsible for the findings.

\section{Conclusions}

An HFrD increased free AA and DHA levels due to membrane degradation as a result of possible oxidative stress and inflammation in the brain. In addition, an HFrD caused histopathological changes in the brain, for example, extensive gliosis, neuronal hydropic degeneration, and edema. In this study, $M$. pruriens had therapeutic effects for FFA metabolism and local inflammatory disorders in the brains of rats fed an HFrD. These findings need to be verified by additional experimental and clinical studies to further contribute to the literature on the therapeutic effects of $M$. pruriens.

\section{Disclosure Statement}

There are no conflicts of interest. 


\section{References}

1 Khitan Z, Kim DH: Fructose: a key factor in the development of metabolic syndrome and hypertension. J Nutr Metab 2013;2013: 682673.

2 Del Zotto H, Borelli MI, Flores L, et al: Islet neogenesis: an apparent key component of long-term pancreas adaptation to increased insulin demand. J Endocrinol 2004;183:321330.

3 Silbernagel G, Machann J, Unmuth S, et al: Effects of 4-week very-high-fructose/glucose diets on insulin sensitivity, visceral fat and intrahepatic lipids: an exploratory trial. $\mathrm{Br} \mathrm{J}$ Nutr 2011;106:79-86.

4 Gerrits PM, Tsalikian E: Diabetes and fructose metabolism. Am J Clin Nutr 1993;58: 796-799.

5 Yilmaz H, Ozcan KS, Sayar N, et al: Metabolic syndrome is associated with atrial electrical and mechanical dysfunction. Med Princ Pract 2015;24:147-152.

6 Chobanian AV, Bakris GL, Black HR, et al: The Seventh Report of the Joint National Committee on Prevention, Detection, Evaluation, and Treatment of High Blood Pressure: the JNC 7 report. JAMA 2003;289:2560-2572.

7 Taha AY, Gao F, Ramadan E, et al: Upregulated expression of brain enzymatic markers of arachidonic and docosahexaenoic acid metabolism in a rat model of the metabolic syndrome. BMC Neurosci 2012;13:131.

8 Woodie L, Blythe S: The differential effects of high-fat and high-fructose diets on physiology and behavior in male rats. Nutr Neurosci 2017;14:1-9.
9 Madani Z, Malaisse WJ, Ait-Yahia D: A comparison between the impact of two types of dietary protein on brain glucose concentrations and oxidative stress in high fructose-induced metabolic syndrome rats. Biomed Rep 2015;3:731-735.

10 Citil C, Konar V, Aydin S, et al: Brain, liver, and serum salusin-alpha and -beta alterations in Sprague-Dawley rats with or without metabolic syndrome. Med Sci Monit 2014;20: 1326-1333.

11 Muller D, Nitsch RM, Wurtman RJ, et al: Streptozotocin increases free fatty acids and decreases phospholipids in rat brain. J Neural Transm 1998;105:1271-1281.

12 Makar TK, Hungund BL, Cook GA, et al: Lipid metabolism and membrane composition are altered in the brains of type II diabetic mice. J Neurochem 1995;64:2159-2168.

13 Majekodunmi SO, Oyagbemi AA, Umukoro $S$, et al: Evaluation of the anti-diabetic properties of Mucuna pruriens seed extract. Asian Pac J Trop Med 2011;4:632-636.

14 Herrera Chalé F, Ruiz Ruiz JC, Betancur Ancona $\mathrm{D}$, et al: The hypolipidemic effect and antithrombotic activity of Mucuna pruriens protein hydrolysates. Food Funct 2016;7: 434-444.

15 Liu W, Ma H, DaSilva NA, et al: Development of a neuroprotective potential algorithm for medicinal plants. Neurochem Int 2016;100: 164-177.

16 Cassani E, Cilia R, Laguna J, et al: Mucuna pruriens for Parkinson's disease: low-cost preparation method, laboratory measures and pharmacokinetics profile. J Neurol Sci 2016;15:365:175-180.
17 Hara A, Radin N.S: Lipid extraction of tissues with a low-toxicity solvent. Anal Biochem 1978;90:420-426.

18 Christie WW: Gas Chromatography and Lipids. Glasgow, The Oily Press, 1992, p 302.

19 Oppelt SA, Zhang W, Tolan DR: Specific regions of the brain are capable of fructose metabolism. Brain Res 2017;1657:312-322.

20 Paoli A, Bianco A, Damiani E, et al: Ketogenic diet in neuromuscular and neurodegenerative diseases. Biomed Res Int 2014;2014: 474296.

21 Agrawal R, Gomez-Pinilla F: "Metabolic syndrome" in the brain: deficiency in omega-3 fatty acid exacerbates dysfunctions in insulin receptor signalling and cognition. J Physiol 2012;590:2485-2499.

22 Yang H, Fan S, Song D, et al: Long-term streptozotocin-induced diabetes in rats leads to severe damage of brain blood vessels and neurons via enhanced oxidative stress. Mol Med Rep 2013;7:431-440.

23 Hristova M, Aloe L: Metabolic syndrome neurotrophic hypothesis. Med Hypotheses 2006;66:545-549.

24 Hotamisligil GS: Inflammation and metabolic disorders. Nature 2006;444:860-867.

25 Bruce-Keller AJ, White CL, Gupta S, et al: NOX activity in brain aging: exacerbation by high fat diet. Free Radic Biol Med 2010;49: 22-30.

26 Souza CG, Moreira JD, Siqueira IR, et al: Highly palatable diet consumption increases protein oxidation in rat frontal cortex and anxiety-like behavior. Life Sci 2007;81:198203 